\title{
Optimality Range of Largest Eigenmode Relaying with Partial Channel State Information in the Relay
}

\author{
Mehdi Molu, Norbert Goertz, \\ Institute of Telecommunications, Vienna University of Technology \\ Email:\{mmortaza, norbert.goertz@nt.tuwien.ac.at\}
}

\begin{abstract}
Optimal precoding in the relay with partial channel state information in the relay is studied in this paper. It is assumed that the antennas of the relay are correlated. Transmission only via the eigenvector corresponding to the largest eigenvalue of the correlation matrix is investigated in this paper which is called Largest Eigenmode Relaying (LER). We derive necessary and sufficient condition under which the transmission achieves capacity only by LER. Although the derived condition requires computationally expensive Monte Carlo simulations to be validated, an alternative method is introduced to circumvent the Monte Carlo simulations: we evaluate a system with infinite number of antennas in the source and derive a closed-form necessary and sufficient condition under which LER is optimal. It is observed, by simulations, that the optimality region of LER is almost independent of the number of antennas in the source; hence, the LER optimality condition derived for infinite number of the antennas in the source can, alternatively, be exploited for systems with finite number of antennas in the source with insignificant difference.
\end{abstract}

\section{INTRODUCTION}

Among various concepts in MIMO communication systems, transmission only via the strongest eigenvector (beamforming) is one of the extensively studied subjects in single-hop MIMO systems. For instance, extensive discussions will be found in [1]-[3] on motivations for studying transmission from the strongest eigenvector.

Recently, there has been a lot of interest for transmission from the strongest eigenvector in multi-hop systems as well [4]-[6]; however, the focus of the literature is, mainly, on beamforming in the source and, hence, there is very less known about beamforming in the relay which is called Maximum Eigenmode Relaying (LER) in this paper.

Two scenarios are described below as motivations to study LER. Assume a user node that relays the information of another node (source node); however, the relay node is unwilling to spend all its power for the transmission of the information belonging to the source node and, hence, as a constraint imposed by the relay node, the relaying is permitted only via the strongest eigenvector. Another motivating example is a scenario where the users are forced to operate at low SNR region in order to mitigate inter-cell interference. In both the aforementioned examples, which are by no means uncommon in practical wireless communication systems, the users (in particular the relay nodes which are of interest to this paper) are forced to operate at a low SNR region where the capacity can be achieved by transmission from the strongest eigenvector. In this paper, the intention is to specify the maximum power that the relay node is allowed to spend while achieving the capacity by transmission from the strongest eigenvector; or alternatively, the intention is to specify the optimal covariance matrix in the relay node in which the capacity can be achieved by transmission from the strongest eigenvector.

\section{Related Work}

A non-coherent cooperative system was investigated in [7], where, the source and the relay nodes are equipped with multiple antennas and the destination node with a single antenna. It was assumed that the source and the relay have access to the covariance matrix of the channels. Another assumption was that the antennas in the source are correlated but no correlation was assumed in the relay. Using that knowledge, the optimal transmit direction in the source and the relay were derived. Assume $Q$ is the transmit covariance matrix in the source with spectral decomposition, $\boldsymbol{Q}=\boldsymbol{U}_{Q} \boldsymbol{\Lambda}_{Q} \boldsymbol{U}_{Q}^{H}$ and $\boldsymbol{\Sigma}$ is the correlation matrix of the source, with spectral decomposition $\boldsymbol{\Sigma}=\boldsymbol{U}_{\Sigma} \boldsymbol{\Lambda}_{\Sigma} \boldsymbol{U}_{\Sigma}^{H}$. It was proved that the optimal $\boldsymbol{Q}$ must be in the form of $\boldsymbol{Q}^{o}=\boldsymbol{U}_{\Sigma} \boldsymbol{\Lambda}_{Q^{\circ}} \boldsymbol{U}_{\Sigma}^{H}$; i.e. $\boldsymbol{U}_{Q^{o}}=\boldsymbol{U}_{\boldsymbol{\Sigma}}$ and $\boldsymbol{\Lambda}_{Q^{\circ}}$ is in descending order which needs to be solved numerically. It was also proved that the optimal gain matrix in the relay is a weighted identity matrix which depends on $n_{\mathrm{R}}, \boldsymbol{\Sigma}$ and $\boldsymbol{Q}$. Furthermore, the necessary and sufficient condition for the optimality of beamforming in the source was derived as

$$
\gamma \lambda_{2}^{\Sigma} \leq \frac{\left(1+\gamma \lambda_{1}^{\Sigma}\right) \mathcal{D}\left(1+\gamma \lambda_{1}^{\Sigma}\right)}{\mathcal{D}\left(1+\gamma \lambda_{1}^{\Sigma}\right)+\mathcal{A}\left(\gamma \lambda_{1}^{\Sigma}\right)-1}-1
$$

where $\mathcal{D}\left(1+\gamma \lambda_{1}^{\Sigma}\right)$ is a function given in [7, Eq. 16] and $\mathcal{A}\left(\gamma \lambda_{1}^{\Sigma}\right)=\mathbb{E}\left\{(1+z) \mathrm{e}^{1+z} \Gamma(0, z)\right\}$ where $\mathbb{E}\{\cdot\}$ represents the expectation operation. Note that the evaluation of the optimality of beamforming in (1) requires computationally expensive Monte Carlo simulations or numerical integrations due to $\mathcal{A}\left(\gamma \lambda_{1}^{\Sigma}\right)$.

Even more recent results were obtained in [8] where an optimal relay precoding was investigated for a system with correlated antennas at the relay. It was assumed that the relay has access to full CSI of the receiving channel, $\boldsymbol{H}_{1}$, but only 
to the covariance matrix of transmit channel, $\boldsymbol{H}_{2}$, i.e. to $\boldsymbol{R}_{\mathrm{R}}$ with spectral decomposition $\boldsymbol{R}_{\mathrm{R}}=\boldsymbol{U}_{\mathrm{R}} \boldsymbol{\Lambda}_{\mathrm{R}} \boldsymbol{U}_{\mathrm{R}}^{H}$. In [8], [9], it was proved that the optimal relay precoding matrix, $\boldsymbol{F}^{o}$, is in the form of $\boldsymbol{F}^{o}=\boldsymbol{U}_{\mathrm{R}} \boldsymbol{\Lambda}_{F}^{o} \boldsymbol{V}_{H_{1}}^{H}$, where $\boldsymbol{V}_{H_{1}}$ is the unitary matrix with columns as the eigenvectors of $\boldsymbol{H}_{1} \boldsymbol{H}_{1}^{H}$. Along with the numerical methods to derive $\Lambda_{F}^{o}$ in [8] , the optimality of beamforming was considered only for the asymptotic case of high transmit SNR. The relay beamforming was found to be optimal if the following inequality holds:

$$
\begin{aligned}
\left(\frac{\sigma^{2}}{P_{\mathrm{R}} \lambda_{1}^{\mathrm{R}}}\right)^{n_{\mathrm{D}}} & \mathrm{e}^{\sigma^{2} / P_{\mathrm{R}} \lambda_{1}^{\mathrm{R}}} \Gamma\left(1-n_{\mathrm{D}}, \frac{\sigma^{2}}{P_{\mathrm{R}} \lambda_{1}^{\mathrm{R}}}\right) \\
& \times\left(\frac{P_{\mathrm{R}} \lambda_{2}^{\mathrm{R}}}{\sigma^{2}}+1\right)+\frac{P_{\mathrm{R}} \lambda_{2}^{\mathrm{R}}}{\sigma^{2}}\left(n_{\mathrm{D}}-1\right) \leq 1
\end{aligned}
$$

For more details, see [8, Sec. III-C].

In this paper, we consider a MIMO cooperative system with multiple antennas at the relay in which the relay has access only to the covariance matrices of its preceding and following channels. Moreover, the relay antennas are assumed to be correlated. With partial channel knowledge (the covariance matrices) available to the relay, a capacity maximizing relaying method will be introduced; the exact system model and the desired performance criterion will be discussed in forthcoming sections.

In [7], it is assumed that the statistical CSI from the sourceto-relay and the relay-to-destination links are available at both the source and the relay nodes but, in this paper, we assume that the source node does not have access to any sort of CSI while the relay node knows about the statistical CSI of its preceding and following channels. Besides, it is assumed in [7] that the relay antennas do not experience fading correlation and, hence, the relay precoder is assumed to be an identity matrix but we assume fading correlation in the relay antennas and, consequently, the relay precoder designed in this paper is non-identity. Note that the optimality of beamforming in the source node is evaluated in [7] while we consider the optimality of beamforming in the relay node, i.e. LER, in this paper. Moreover, verification of the optimality of beamforming in the source node in [7] requires computationally expensive Monte Carlo simulations but in this paper, apart from the approach which requires Monte Carlo simulations, we propose a tight closed-form solution for the verification of the optimality of LER. The problem considered in this paper is similar to [8]: a relay precoder is designed in both the papers but the difference is that we assume statistical CSI from the source-to-relay link at the relay but in [8], perfect CSI from the source-to-relay link is assumed. Moreover, in order to alleviate the mathematical complexity, the optimality of LER is considered only for high SNR regime in [8], but we consider the optimality of LER for entire SNR regime.

The paper is organized as follows: In Section II, the system model is explained and two problems are stated that are the main subject of this paper. Section III deals with designing optimal precoding in the relay in order to maximise capacity. In Section IV, the necessary and sufficient conditions for the optimality of LER are investigated. In Section V some asymptotic results and their usefulness in practical systems are considered. In Section VI numerical results are presented and finally, some conclusion remarks are explained in Section VII.

\section{System Model and Problem Statement}

\section{Notation}

Matrices are represented by boldface upper cases $(\boldsymbol{H})$. Column and row vectors are denoted by boldface lower cases ( $\boldsymbol{h}$ ), and $h_{i}$ indicates the $i$-th element of $\boldsymbol{h}$. The superscript ${ }^{H}$ stands for Hermitian transposition. We refer to the identity matrix by $\boldsymbol{I}$. The expectation operation is indicated by $\mathbb{E}\{\cdot\}$ and $f_{X}(x)$ is reserved for probability density functions (pdf) of random variable $X ; \boldsymbol{\Lambda}_{\Sigma}$ represents a diagonal matrix with elements organized in descending order and $\lambda_{i}^{\Sigma}$ denotes the $i$ th diagonal element of $\boldsymbol{\Lambda}_{\Sigma}$. For simplicity of notation, $\left(\lambda_{i}^{\Sigma}\right)^{2}$ is abbreviated by $\lambda_{i}^{\Sigma 2}$. The trace of a matrix is denoted by $\operatorname{Tr}(\cdot)$.

\section{A. System Model}

A dual hop, half duplex non-coherent MIMO communication system is considered in this paper. A source node with $n_{\mathrm{S}}$ antennas communicates with a single-antenna destination node only via a relay node that is equipped with $n_{\mathrm{R}}$ antennas (that are used for both reception and transmission). It is assumed that a direct link between the source and the destination is not available. The half duplex constraint is accomplished by time sharing between the source and the relay; i.e. each transmission period is divided into two time slots: the source transmits during the first time slot and the relay during the second one. The relay remains silent during the source transmission and vice versa. It is assumed that the source does not have access to any statistical or instantaneous channel state information (CSI). The signal received at the relay $\left(\boldsymbol{y}_{\mathrm{R}}\right)$ due to the source transmission is given by

$$
\boldsymbol{y}_{\mathrm{R}}=\boldsymbol{H}_{1} \boldsymbol{x}+\boldsymbol{w}_{\mathrm{R}}
$$

where the $n_{\mathrm{R}} \times n_{\mathrm{S}}$ matrix $\boldsymbol{H}_{1}$ represents channel between the source and the relay (below, only the statistics of $\boldsymbol{H}_{1}$ are assumed to be known at the relay). With $P_{\mathrm{S}}$ the power constraint of the source, the column vector $\boldsymbol{x}$ is the signal transmitted from the source with $\boldsymbol{Q}=\mathbb{E}\left(\boldsymbol{x} \boldsymbol{x}^{H}\right)=\frac{P_{\mathrm{S}}}{n_{\mathrm{S}}} \boldsymbol{I}_{n_{\mathrm{S}}}$ and the column vector $\boldsymbol{w}_{\mathrm{R}}$ represents the receiver noise in the relay with elements independently drawn from a complex Gaussian random variable with variance $N_{0}$. The relay multiplies $\boldsymbol{y}_{\mathrm{R}}$ with gain matrix $\boldsymbol{F}$ and forwards it to the destination. Then, the received signal at the destination is

$$
\begin{aligned}
\boldsymbol{y}_{\mathrm{D}} & =\boldsymbol{h}_{2} \boldsymbol{F} \boldsymbol{y}_{\mathrm{R}}+\boldsymbol{w}_{\mathrm{D}} \\
& =\boldsymbol{h}_{2} \boldsymbol{F} \boldsymbol{H}_{1} \boldsymbol{x}+\boldsymbol{h}_{2} \boldsymbol{F} \boldsymbol{w}_{\mathrm{R}}+\boldsymbol{w}_{\mathrm{D}}
\end{aligned}
$$

where the row vector $\boldsymbol{h}_{2}$ indicates the channel between the relay and the destination; $\boldsymbol{w}_{\mathrm{D}}$ represents the receiver noise at the destination. For simplicity, we assume that $\boldsymbol{w}_{\mathrm{D}}$ is statistically equivalent to $\boldsymbol{w}_{\mathrm{R}}$ and that both noise processes have unit-variance, i.e., $N_{0}=1$; the latter choice is no extra 
restriction, as the ratios of transmit powers and noise powers determine performance, and we are still free to choose $P_{\mathrm{S}}$ and $P_{\mathrm{R}}$ arbitrarily.

We assume spatial correlation only at the relay, which can be due to unobstructed relay node or space limits at the relay which forces antennas to be closely located. Justifications to assume transceivers with spatial correlation can be found in [10], [11]. The correlation matrix in the relay is represented by $\boldsymbol{\Sigma}$ with spectral decomposition $\boldsymbol{\Sigma}=\boldsymbol{U}_{\Sigma} \boldsymbol{\Lambda}_{\Sigma} \boldsymbol{U}_{\Sigma}^{H}$, where $\boldsymbol{U}_{\Sigma}$ is a unitary matrix with its columns the eigenvectors corresponding to $\boldsymbol{\Sigma}$, and $\boldsymbol{\Lambda}_{\Sigma}$ is diagonal matrix with the eigenvalues of $\boldsymbol{\Sigma}$ in decreasing order. Note that we assume i.i.d. channels for $\boldsymbol{H}_{1}$ and $\boldsymbol{h}_{2}$. Therefore, the channel matrices $\boldsymbol{H}_{1}$ and $\boldsymbol{h}_{2}$ can be written using the Kronecker model as

$$
\begin{aligned}
\boldsymbol{H}_{1} & =\boldsymbol{\Sigma}^{\frac{1}{2}} \boldsymbol{H}_{1 w} \\
\boldsymbol{h}_{2} & =\boldsymbol{h}_{2 w} \boldsymbol{\Sigma}^{\frac{1}{2}}
\end{aligned}
$$

where $\boldsymbol{H}_{1 w}$ and $\boldsymbol{h}_{2 w}$ are i.i.d., zero mean, unit variance complex Gaussian random variables, independent of each other; $\boldsymbol{\Sigma}^{\frac{1}{2}}$ denotes a matrix formed by the square roots of the elements in the matrix $\boldsymbol{\Sigma}$.

\section{B. Problem Statement}

With (4), the ergodic capacity of the system is defined as

$$
C=\frac{1}{2} \max _{\substack{\boldsymbol{Q}=\frac{P_{\mathrm{S}}}{n_{\mathrm{S}}} \boldsymbol{I} \\ F: \mathbb{E}\left\{\left\|\boldsymbol{F} \boldsymbol{y}_{\mathrm{R}}\right\|^{2}\right\} \leq P_{\mathrm{R}}}} \mathbb{E}\left\{C\left(\boldsymbol{H}_{1}, \boldsymbol{h}_{2}, \boldsymbol{F}\right)\right\}
$$

where the relay gain matrix $\boldsymbol{F}$ is to be designed to maximise (7), when the expectation operation is carried out over $\boldsymbol{H}_{1}$ and $\boldsymbol{h}_{2}$. Assuming $\boldsymbol{Q}=\frac{P_{\mathrm{S}}}{n_{\mathrm{S}}} \boldsymbol{I}_{n_{\mathrm{S}}}$ (this means equal transmit power from each antenna is chosen in the source, because no channel knowledge is available there), the channel capacity $C\left(\boldsymbol{H}_{1}, \boldsymbol{h}_{2}, \boldsymbol{F}\right)$ for given channel matrices is

$$
C\left(\boldsymbol{H}_{1}, \boldsymbol{h}_{2}, \boldsymbol{F}\right)=\log \left(1+\frac{P_{\mathrm{S}}}{n_{\mathrm{S}}} \frac{\boldsymbol{h}_{2} \boldsymbol{F} \boldsymbol{H}_{1} \boldsymbol{H}_{1}^{H} \boldsymbol{F}^{H} \boldsymbol{h}_{2}^{H}}{N_{0}\left(1+\boldsymbol{h}_{2} \boldsymbol{F} \boldsymbol{F}^{H} \boldsymbol{h}_{2}^{H}\right)}\right)
$$

where $N_{0}\left(1+\boldsymbol{h}_{2} \boldsymbol{F} \boldsymbol{F}^{H} \boldsymbol{h}_{2}^{H}\right)$ is the total equivalent noise power which remains constant per coherence time due to a block fading assumption we impose.

Two major problems are addressed below:

Problem 1: Solving the optimization problem in (7), in order to find the optimal precoder $\boldsymbol{F}^{o}$ in the relay that maximizes mutual information between the transmit signal from the source and the received signal at the destination, given that the correlation matrix $\boldsymbol{\Sigma}$ as well as the unit variance of elements of i.i.d $\boldsymbol{H}_{1 w}$ and $\boldsymbol{h}_{2 w}$ are available at the relay.

Problem 2: Obtaining necessary and sufficient conditions based on $\Sigma$, so that maximum eigenmode relaying (LER) is optimal.

\section{Optimal PRECODING IN THE RELAY}

In this section, Problem 1 from Section II-B is presented without proof due to space limit. However, the proof to design optimal $\boldsymbol{F}$ follows similar steps as in [7] or [9].
It can be proved that $\boldsymbol{F}$ is a positive symmetric matrix and $\boldsymbol{F}=\boldsymbol{G}^{\frac{1}{2}}$ with spectral decomposition $\boldsymbol{G}=\boldsymbol{U}_{G} \boldsymbol{\Lambda}_{G} \boldsymbol{U}_{G}^{H}$. Assuming spectral decomposition $\boldsymbol{\Sigma}=\boldsymbol{U}_{\Sigma} \boldsymbol{\Lambda}_{\Sigma} \boldsymbol{U}_{\Sigma}^{H}$, it can be proved that the optimal $\boldsymbol{G}$ must direct the transmission from the relay in the direction of eigenvectors of $\boldsymbol{\Sigma}$, i.e. $\boldsymbol{U}_{G}=\boldsymbol{U}_{\Sigma}$ must hold. Consequently, it dictates that $\boldsymbol{G}=\boldsymbol{U}_{\Sigma} \boldsymbol{\Lambda}_{G} \boldsymbol{U}_{\Sigma}^{H}$ where $\Lambda_{G}$ is to be determined using numerical methods (see e.g. [12] for a single hop system model).

\section{Optimality of Maximum Eigenmode Relaying}

In this Section, Problem 2 from Section II-B is addressed. The focus of this section is to derive necessary and sufficient conditions under which LER is the optimal transmission method from the relay.

By the results obtained in Section III, the ergodic capacity in (7) can be rewritten as

$$
\begin{aligned}
& C=\frac{1}{2} \max _{\substack{\boldsymbol{Q}=\frac{P_{\mathrm{S}}}{n_{\mathrm{S}}} \boldsymbol{I} \\
\boldsymbol{\Lambda}_{G}: P_{\mathrm{S}} \operatorname{Tr}\left(\boldsymbol{\Lambda}_{\Sigma} \boldsymbol{\Lambda}_{G}\right)+N_{0} \operatorname{Tr}\left(\boldsymbol{\Lambda}_{G}\right) \leq P_{\mathrm{R}}}} \mathbb{E}\left\{C\left(\boldsymbol{H}_{1 w}, \boldsymbol{h}_{2 w}, \boldsymbol{\Lambda}_{G}\right)\right\}
\end{aligned}
$$

where the second constraint $\boldsymbol{\Lambda}_{G}: \cdots \leq P_{\mathrm{R}}$ is obtained by some manipulations on $\mathbb{E}\left\{\left\|\boldsymbol{F} \boldsymbol{y}_{\mathrm{R}}\right\|^{2}\right\}$ in (7). The expectationoperation is carried out over $\boldsymbol{H}_{1 w}$ and $\boldsymbol{h}_{2 w}$. From (9), $C\left(\boldsymbol{H}_{1 w}, \boldsymbol{h}_{2 w}, \boldsymbol{\Lambda}_{G}\right)$ is

$$
\begin{aligned}
C\left(\boldsymbol{H}_{1 w}, \boldsymbol{h}_{2 w}, \boldsymbol{\Lambda}_{G}\right) & = \\
& \log \left(1+\frac{\gamma \boldsymbol{h}_{2 w} \boldsymbol{\Lambda}_{G}^{\frac{1}{2}} \boldsymbol{\Lambda}_{\Sigma} \boldsymbol{H}_{1 w} \boldsymbol{H}_{1 w}^{H} \boldsymbol{\Lambda}_{G}^{\frac{1}{2}} \boldsymbol{\Lambda}_{\Sigma} \boldsymbol{h}_{2 w}^{H}}{n_{\mathrm{S}}\left(1+\boldsymbol{h}_{2 w} \boldsymbol{\Lambda}_{G} \boldsymbol{\Lambda}_{\Sigma}^{2} \boldsymbol{h}_{2 w}^{H}\right)}\right)
\end{aligned}
$$

with $\gamma=P_{\mathrm{S}} / N_{0}$ and the assumption that product of a unitary matrix with a random matrix does not change the distribution of the random matrix. Then $C\left(\boldsymbol{H}_{1 w}, \boldsymbol{h}_{2 w}, \boldsymbol{\Lambda}_{G}\right)$ can be simplified according to

$$
\begin{aligned}
& C\left(\boldsymbol{H}_{1 w}, \boldsymbol{h}_{2 w}, \boldsymbol{\Lambda}_{G}\right)= \\
& \log \left(1+\frac{\gamma \sum_{i=1}^{n_{\mathrm{S}}}\left|\boldsymbol{h}_{2 w} \boldsymbol{\Lambda}_{G}^{\frac{1}{2}} \boldsymbol{\Lambda}_{\Sigma} \boldsymbol{h}_{1 w, i}\right|^{2}}{n_{\mathrm{S}}\left(1+\boldsymbol{h}_{2 w} \boldsymbol{\Lambda}_{G} \boldsymbol{\Lambda}_{\Sigma}^{2} \boldsymbol{h}_{2 w}^{H}\right)}\right)
\end{aligned}
$$

where $\boldsymbol{h}_{1 w, i}$ represents the $i$ th column of $\boldsymbol{H}_{1 w}$. One can write for the numerator

$$
\frac{1}{n_{\mathrm{S}}} \sum_{i=1}^{n_{\mathrm{S}}}\left|\boldsymbol{h}_{2 w} \boldsymbol{\Lambda}_{G}^{\frac{1}{2}} \boldsymbol{\Lambda}_{\Sigma} \boldsymbol{h}_{1 w, i}\right|^{2}=\sum_{j=1}^{n_{\mathrm{R}}} \lambda_{j}^{G} \lambda_{j}^{\Sigma 2} X_{j} Y
$$

where $X_{j}=\left|h_{2 w, j}\right|^{2}$ is an exponential random variable with unit mean, i.e. $f_{X_{j}}(t)=\mathrm{e}^{-t}$, and $Y=\frac{1}{n_{\mathrm{S}}} \sum_{i=1}^{n_{\mathrm{s}}}\left|h_{1 w, i}\right|^{2}$ is the sum of $n_{\mathrm{S}}$ i.i.d exponential random variables with parameter $n_{\mathrm{S}}$. Indeed, $Y$ has an Erlang-distribution with rate and shape equal to $n_{\mathrm{S}}$. Proof is omitted due to space limit. Likewise, for the denominator of (11), one can write

$$
\boldsymbol{h}_{2 w} \boldsymbol{\Lambda}_{G} \boldsymbol{\Lambda}_{\Sigma}^{2} \boldsymbol{h}_{2 w}^{H}=\sum_{j=1}^{n_{\mathrm{R}}} \lambda_{j}^{G} \lambda_{j}^{\Sigma 2} X_{j}
$$

Plugging in (12) and (13) into (11) and basic algebraic 
manipulations, (11) will further simplify to

$$
\begin{aligned}
C\left(\boldsymbol{H}_{1 w}, \boldsymbol{h}_{2 w}, \boldsymbol{\Lambda}_{G}\right)= & \log \left(1+\sum_{j=1}^{n_{\mathrm{R}}} \lambda_{j}^{G} \lambda_{j}^{\Sigma 2}(1+\gamma Y) X_{j}\right) \\
& -\log \left(1+\sum_{j=1}^{n_{\mathrm{R}}} \lambda_{j}^{G} \lambda_{j}^{\Sigma 2} X_{j}\right) .
\end{aligned}
$$

\section{A. Necessary Condition}

Now we turn the attention to the question under which condition LER is optimal; i.e. the condition under which $\lambda_{j}^{G}=0$ for $j \geq 2$ is the capacity achieving transmission method with

$$
\lambda_{1}^{G}=\frac{P_{\mathrm{R}}}{\left(N_{0}+\lambda_{1}^{\Sigma} P_{\mathrm{S}}\right)}
$$

which is calculated by substituting $\lambda_{j}^{G}=0$ for $j \geq 2$ in $P_{\mathrm{S}} \operatorname{Tr}\left(\boldsymbol{\Lambda}_{\Sigma} \boldsymbol{\Lambda}_{G}\right)+N_{0} \operatorname{Tr}\left(\boldsymbol{\Lambda}_{G}\right)=P_{\mathrm{R}}$, from the second constraint in (9).

In order to evaluate the necessary and sufficient condition on the optimality of LER, we assume $P=\sum_{j=1}^{n_{\mathrm{R}}} \lambda_{j}^{G}$. Assume that the power $P-p$ is allocated to the dominant eigenvector of $G$, i.e. $\lambda_{1}^{G}=P-p$, and the power $p$ is allocated to the remaining eigenvectors of $\boldsymbol{G}$, i.e. $p=\sum_{j=2}^{n_{\mathrm{R}}} \lambda_{j}^{G}$. It is clear that if LER is optimal, then $\partial C(p) /\left.\partial p\right|_{p=0} \leq 0$ which provides the necessary condition for the optimality of LER and $\partial^{2} C(p) /\left.\partial p^{2}\right|_{p=0} \leq 0$ which confirms the sufficiency. It can be proved that $\partial C(p) /\left.\partial p\right|_{p=0}$ will be maximized if $\lambda_{2}^{G}=p$ and $\lambda_{j}^{G}=0$ for $j>2$; similar discussions are provided in [1], [2], [7] and, hence, we do not repeat it here. Therefore, given the assumption $\lambda_{1}^{G}=P-p$ and $\lambda_{2}^{G}=p$, (14) will be further simplified to

$$
\begin{aligned}
C(p)= & \log \left(1+(P-p) \lambda_{1}^{\Sigma 2}(1+\gamma Y) X_{1}+p \lambda_{2}^{\Sigma 2}(1+\gamma Y) X_{2}\right) \\
& -\log \left(1+(P-p) \lambda_{1}^{\Sigma 2} X_{1}+p \lambda_{2}^{\Sigma 2} X_{2}\right)
\end{aligned}
$$

and so, $\partial C(p) /\left.\partial p\right|_{p=0}$ from (9) and (16) equals

$$
\begin{aligned}
\left.\frac{\partial C(p)}{\partial p}\right|_{p=0}= & \mathbb{E}\left\{\frac{\lambda_{2}^{\Sigma 2}(1+\gamma Y) X_{2}}{1+P \lambda_{1}^{\Sigma 2}(1+\gamma Y) X_{1}}\right\} \\
& -\mathbb{E}\left\{\frac{\lambda_{1}^{\Sigma 2}(1+\gamma Y) X_{1}}{1+P \lambda_{1}^{\Sigma 2}(1+\gamma Y) X_{1}}\right\} \\
& -\mathbb{E}\left\{\frac{\lambda_{2}^{\Sigma 2} X_{2}-\lambda_{1}^{\Sigma 2} X_{1}}{1+P \lambda_{1}^{\Sigma 2} X_{1}}\right\} .
\end{aligned}
$$

Assuming $Z=1+P \lambda_{1}^{\Sigma 2}(1+\gamma Y) X_{1}$, the second expectation on the right hand side of (17) is

$$
\mathbb{E}\left\{\frac{\lambda_{1}^{\Sigma 2}(1+\gamma Y) X_{1}}{Z}\right\}=\frac{1}{P}-\frac{1}{P} \mathbb{E}\left\{\frac{1}{Z}\right\}
$$

and the last expectation in (17) can be written as

$\mathbb{E}\left\{\frac{\lambda_{2}^{\Sigma 2} X_{2}-\lambda_{1}^{\Sigma 2} X_{1}}{1+P \lambda_{1}^{\Sigma 2} X_{1}}\right\}=\frac{e^{\frac{1}{P \lambda_{1}^{\Sigma 2}}}\left(1+P \lambda_{2}^{\Sigma 2}\right) \Gamma\left(0, \frac{1}{P \lambda_{1}^{\Sigma 2}}\right)}{P^{2} \lambda_{1}^{\Sigma 2}}-\frac{1}{P}$

where $\Gamma(0, z)$ is the incomplete Gamma function [13].

By combining (9), (17), (18) and (19), in order to compute $\partial C(p) /\left.\partial p\right|_{p=0} \leq 0$, we have

$$
\begin{aligned}
\left.\frac{\partial C(p)}{\partial p}\right|_{p=0}= & \mathbb{E}\left\{\frac{\lambda_{2}^{\Sigma 2}(1+\gamma Y)+1 / P}{1+P \lambda_{1}^{\Sigma 2}(1+\gamma Y) X_{1}}\right\} \\
& -\frac{\mathrm{e}^{\frac{1}{P \lambda_{1}^{\Sigma 2}}}\left(1+P \lambda_{2}^{\Sigma 2}\right) \Gamma\left(0, \frac{1}{P \lambda_{1}^{\Sigma 2}}\right)}{\left(P \lambda_{1}^{\Sigma}\right)^{2}} \leq 0
\end{aligned}
$$

from which, by elementary operations, the following constraint will be obtained for the LER to be optimal:

$$
\lambda_{2}^{\Sigma 2} \leq \frac{\mathrm{e}^{\frac{1}{P \lambda_{1}^{\Sigma 2}}} \Gamma\left(0, \frac{1}{P \lambda_{1}^{\Sigma 2}}\right)-P \lambda_{1}^{\Sigma 2} \mathbb{E}\left\{\frac{1}{Z}\right\}}{P^{2} \lambda_{1}^{\Sigma 2} \mathbb{E}\left\{\frac{(1+\gamma Y)}{Z}\right\}-P \mathrm{e}^{\frac{1}{P \lambda_{1}^{\Sigma 2}}} \Gamma\left(0, \frac{1}{P \lambda_{1}^{\Sigma 2}}\right)}
$$

Note that $\mathbb{E}\{1 / Z\}$ and $\mathbb{E}\{(1+\gamma Y) / Z\}$ in (21) are non-trivial and do not seem to have closed-form solutions. However, they can be calculated either using Monte Carlo simulations or by numerical integration.

\section{B. Sufficient Condition}

By deriving the necessary condition for LER to be optimal, it can be proved that the necessary condition is also a sufficient condition for LER to be optimal. It will be proved by showing that $\partial^{2} C(p) / \partial p^{2} \leq 0$ for arbitrary $p \in[0, P]$ where $\lambda_{1}^{G}=P-p, \lambda_{2}^{G}=p$ and $\lambda_{i}^{G}=0$ for $i>2$.

It is straightforward to derive $\partial^{2} C(p) / \partial p^{2}$ from (9) and (16) as follows

$$
\begin{aligned}
& \left.\frac{\partial^{2}}{\partial p^{2}} \mathbb{E}\left\{C\left(\boldsymbol{H}_{1 w}, \boldsymbol{h}_{2 w}, \boldsymbol{\Lambda}_{G}\right)\right\}\right|_{p=0}=-P_{\mathrm{S}} Y \\
& \quad \times \frac{\left(\lambda_{1}^{\Sigma 2} X_{1}-\lambda_{2}^{\Sigma 2} X_{2}\right)^{2}\left(2+\gamma Y+2 P \lambda_{1}^{\Sigma 2} X_{1}(1+\gamma Y)\right)}{n_{\mathrm{S}} N_{0}\left(1+P \lambda_{1}^{\Sigma 2} X_{1}\right)^{2}\left(1+P\left(\lambda_{1}^{\Sigma}\right)^{2} X_{1}(1+\gamma Y)\right)^{2}}
\end{aligned}
$$

It is clear from (22) that $\partial^{2} C(p) / \partial p^{2} \leq 0$ for every $p$, and so, the necessary condition for the optimality of LER derived in (21) is sufficient as well.

\section{LARge Antenna ARray In the Source}

In the previous section, a necessary and sufficient condition was derived which shows the optimality region of LER. It is clear from (20) or (21) that there is a need to perform Monte Carlo simulations or numerical integrations to validate the optimality of LER. However, computationally expensive Monte Carlo simulations and also numerical integration methods are not a good choice in practical real-time communication systems; therefore, in the sequel, we will investigate a simplified approach which lead to closed-form solutions.

We investigate the effect of the source antenna array on the optimality of LER. That is done by considering large antenna arrays in the source using the central limit theorem. Novel closed-form necessary and sufficient condition is derived for the optimality of LER which, according to the simulations, turn out to very tightly approximate the results for arbitrary numbers of antennas in the source.

The random variable $Y$ in the previous sections, for instance in equations (12) or (21), follows an Erlang-distribution; indeed, $Y$ is the sum of $n_{\mathrm{S}}$ exponential random variables, 


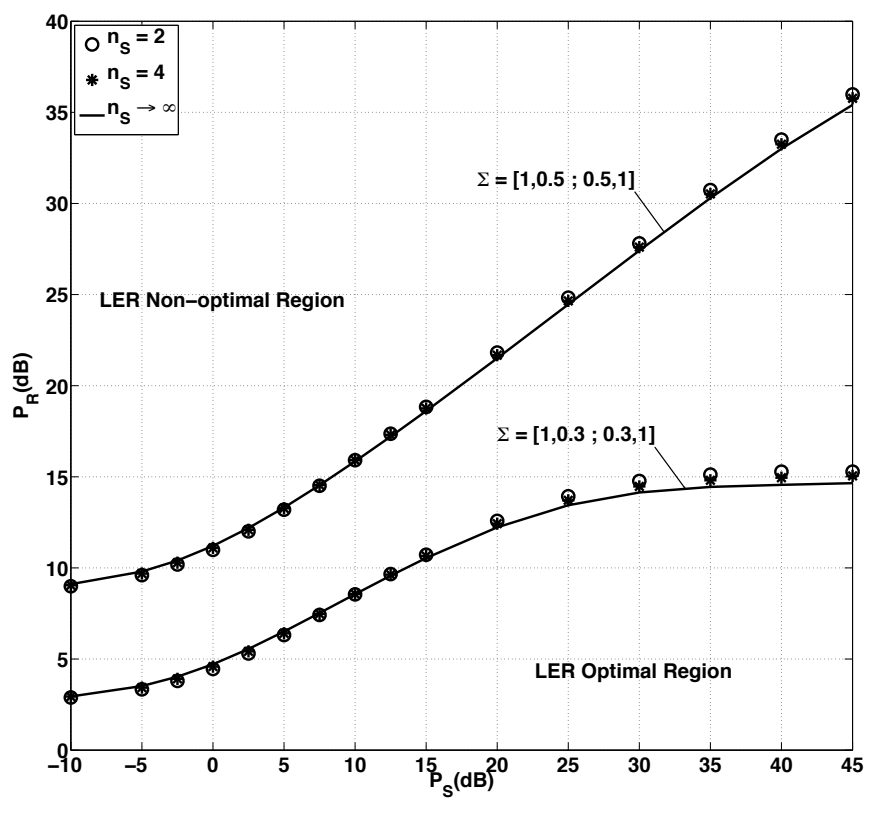

Fig. 1. LER optimality region for $\left(P_{\mathrm{S}}, P_{\mathrm{R}}\right)$ pairs for various correlations $\Sigma$ and assuming $N_{0}=1$.

each with parameter $n_{\mathrm{S}}$. Using central limit theorem (See [14, Ch. 7.4]), the random variable $Y$ can be approximated by a Gaussian distribution with mean equal to 1 and variance $1 / n_{\mathrm{S}}$, i.e., approximately, $Y \sim \mathcal{N}\left(1,1 / n_{\mathrm{S}}\right)$. Let Assume $n_{\mathrm{S}} \gg 1$, therefore, as $n_{\mathrm{S}} \rightarrow \infty$, we obtain $Y \rightarrow 1$. By substituting $Y=1$ in (21), we have $1+\gamma Y=1+\gamma$. Hence, $\mathbb{E}\{1 / Z\}$ in (21) can be written in closed-form as

$$
\mathbb{E}\left\{\frac{1}{Z}\right\}=\mathcal{A} \mathrm{e}^{\mathcal{A}} \Gamma(0, \mathcal{A})
$$

where $\mathcal{A}=1 / P \lambda_{1}^{\Sigma 2}(1+\gamma)$. Then, the necessary and sufficient conditions for the optimality of LER, i.e. $\partial C(p) /\left.\partial p\right|_{p=0} \leq 0$ in (20) or (21) for large $n_{\mathrm{S}}$, after some manipulation lead to the following closed-form constraint:

$$
\lambda_{2}^{\Sigma 2} \leq \frac{\left.\mathrm{e}^{\frac{1}{P \lambda_{1}^{\Sigma 2}}} \Gamma\left(0, \frac{1}{P \lambda_{1}^{\Sigma 2}}\right)-P \lambda_{1}^{\Sigma 2} \mathcal{A} \mathrm{e}^{\mathcal{A}} \Gamma(0, \mathcal{A})\right\}}{P \mathrm{e}^{\mathcal{A}} \Gamma(0, \mathcal{A})-P \mathrm{e}^{\frac{1}{P \lambda_{1}^{\Sigma 2}}} \Gamma\left(0, \frac{1}{P \lambda_{1}^{\Sigma 2}}\right)} .
$$

It is clear from (24) that with large antenna array in the source, the optimality of LER depends on the source transmission only via $P_{\mathrm{S}}$ and it is independent of $\boldsymbol{H}_{1}$.

It will be shown, by numerical simulations in the next Section, that the closed-form constraint in (24) approximates (21) with high accuracy.

\section{NUMERICAL RESUlTS}

We assume that the relay is equipped with two antennas, but various numbers of the antennas in the source are evaluated. As explained in Section II, we assume i.i.d $\boldsymbol{H}_{1, w}$ and $\boldsymbol{h}_{2, w}$, where the elements are circularly symmetric complex Gaussian random variables with zero mean and unit variance (block
Rayleigh fading assumption). The noise power in the relay and the destination is assumed to be $N_{0}=1$. Assuming $n_{\mathrm{S}}=2$ and 4 , Fig. 1 illustrates the $\left(P_{\mathrm{S}}, P_{\mathrm{R}}\right)$-pairs for which LER is optimal. The optimality region of LER is calculated for $\rho=0.3$ and 0.5 where $\rho$ is defined as the inter-antenna correlation or the off-diagonal elements of the correlation matrix $\Sigma$. It is clear from Fig. 1 that by increasing $\rho$, the optimality region of LER increases, too. It is clear from Fig. 1 that the closed-form constraint (24) tightly approximates (21) which requires Monte Carlo simulations. It explicitly shows that regardless of $n_{\mathrm{S}}$, the optimal regions of LER for various $n_{\mathrm{S}}$ coincide with very little difference.

\section{CONCLUSION}

A dual hop MIMO cooperative system was investigated in this paper. It was assumed that the antennas of the relay are correlated. A Necessary and sufficient conditions was derived under which the transmission from the largest eigenmode in the relay achieves capacity.

\section{REFERENCES}

[1] S. Jafar, S. Vishwanath, and A. Goldsmith, "Channel capacity and beamforming for multiple transmit and receive antennas with covariance feedback," in IEEE International Conference on Communications (ICC), vol. 7, pp. 2266-2270 vol.7, June 2001.

[2] E. Visotsky and U. Madhow, "Space-time transmit precoding with imperfect feedback," IEEE Transactions on Information Theory, vol. 47, pp. 2632-2639, Sept. 2001.

[3] X. Li, S. Jin, X. Gao, and K.-K. Wong, "Near-optimal power allocation for MIMO channels with mean or covariance feedback," IEEE Transactions onCommunications, vol. 58, pp. 289 -300, january 2010.

[4] C. Zhong, T. Ratnarajah, S. Jin, and K.-K. Wong, "Performance analysis of optimal single stream beamforming in MIMO dual-hop AF systems," IEEE Journal on Selected Areas in Communications, vol. 30, pp. 1415 -1427 , september 2012

[5] M. Li, M. Lin, Q. Yu, W.-P. Zhu, and L. Dong, "Optimal beamformer design for dual-hop MIMO AF relay networks over rayleigh fading channels," IEEE Journal onSelected Areas in Communications, vol. 30, pp. $1402-1414$, september 2012

[6] L. Sanguinetti, A. D'Amico, and Y. Rong, "A tutorial on the optimization of amplify-and-forward MIMO relay systems," IEEE Journal on Selected Areas in Communications, vol. 30, pp. 1331 -1346, september 2012.

[7] P. Dharmawansa, M. McKay, R. Mallik, and K. Ben Letaief, "Ergodic capacity and beamforming optimality for multi-antenna relaying with statistical CSI," IEEE Transactions on Communications, vol. 59, pp. 2119-2131, Aug. 2011.

[8] C. Jeong, B. Seo, S. R. Lee, H.-M. Kim, and I.-M. Kim, "Relay precoding for non-regenerative MIMO relay systems with partial CSI feedback," IEEE Transactions on Wireless Communications, vol. 11, pp. 1698-1711, May 2012.

[9] C. Jeong and H.-M. Kim, "Precoder design of non-regenerative relays with covariance feedback," IEEE Communications Letters, vol. 13 pp. 920-922, Dec. 2009.

[10] D.-S. Shiu, G. Foschini, M. Gans, and J. Kahn, "Fading correlation and its effect on the capacity of multielement antenna systems," IEEE Transactions on Communications, vol. 48, pp. 502-513, Mar. 2000.

[11] D. shan Shiu, Wireless Communication Using Dual Antenna Arrays. Kluwer Academic Publishers, Nov. 1999.

[12] E. Jorswieck and H. Boche, "Optimal transmission with imperfect channel state information at the transmit antenna array," Wireless Personal Communication, vol. 27, pp. 33-56, Jan. 2003.

[13] Abramowitz and Stegun, Handbook of Mathematical Functions. Dover Publications, June 1965.

[14] P. A. and P. S.U., Probability, Random Variables and Stochastic Processes. McGraw Hill, 4th ed., 2002. 